\title{
Towards microsystems for automatic acquisition of in vivo gastrointestinal information
}

\author{
Katsuya Morimoto, Shigeki Yamaguchi, Junji Fukuda, and Hiroaki Suzuki ${ }^{\text {a) }}$ \\ Graduate School of Pure and Applied Sciences, University of Tsukuba, 1-1-1 Tennodai, Tsukuba, Ibaraki 305- \\ 8573, Japan
}

(Received 31 March 2008; accepted 8 July 2008; published online 19 May 2009)

\begin{abstract}
The possibility to develop a microanalysis system for the acquisition of gastrointestinal information is presented here. The system consists of four assay sites for trypsin, pepsin, and other biochemical compounds. The major components in each assay site were a $p \mathrm{H}$-responsive valve, a $p \mathrm{H}$-stat used to maintain the $p \mathrm{H}$ of the solution to be analyzed and used for electrochemical $p \mathrm{H}$-titration, and a freeze-dried enzyme substrate stored in the $p \mathrm{H}$-stat. The operation of the valve is based on electrowetting, and the valve is made $p \mathrm{H}$-responsive by means of a nonstandard three-electrode system. The sample solution was automatically injected into the compartment and rapidly dissolved into the substrate layer. The automatic $p \mathrm{H}$-stat, based on another nonstandard use of the electrochemical three-electrode system, maintained the solution $p \mathrm{H}$ and, at the same time, conducted $p \mathrm{H}$-titration. The determination of the activity of the proteases was conducted at their optimum $p$ Hs. The output current showed a clear dependence on the activity of the enzymes. Integrating the functions provides significant advantages for the use of this system as an isolated telemetric microsystem that may operate with wireless signal transmission using a small power supply. (C) 2009 American Institute of Physics. [DOI: 10.1063/1.3116086]
\end{abstract}

\section{INTRODUCTION}

Disorders relating to the in vivo activity of digestive enzymes, such as trypsin and pepsin, are known to be related to gastrointestinal diseases. For example, some pancreatic diseases, including acute pancreatitis and cystic fibrosis, are accompanied with an increase in the activity of trypsin. ${ }^{1,2}$ Trypsin plays an important role in activating all other pancreatic proenzymes within the intestine, thereby controlling pancreatic exocrine functions.

Recently, progress in micrototal analysis systems or labon-a-chip technology has produced promising devices for health care or point-of-care testing. Although the realized forms are diverse and the applicable fields are rapidly expanding, capsule-type microsystems that directly collect gastrointestinal information in vivo will be of great help for the accurate diagnoses of gastrointestinal diseases and fundamental gastroenterology and are expected to revolutionize clinical diagnosis. ${ }^{3}$ The devices will have no wires connecting them to controlling instruments, and the information will be conveyed by wireless transmission. Although several attempts have been made to construct systems for the acquisition of physical information, ${ }^{4,5}$ the incorporation of sensing functions for biochemical analytes has just begun. ${ }^{6}$

Of the basic functions required for enzyme analyses, we focused our attention on sampling, mixing of components, $p \mathrm{H}$-regulation, and sensing. In developing devices for use in an isolated environment, the components should preferably function automatically. Although a considerable amount of time will be required to develop ideal devices, researchers

\footnotetext{
a) Author to whom correspondence should be addressed. Tel.: +81-29-853-5598. FAX: +81-29-853-4490. Electronic mail: hsuzuki@ims.tsukuba.ac.jp.
}

are making steady progress toward this goal. Our approach was to take full advantage of electrochemical techniques. A simple microvalve can be developed based on eletrowetting. By incorporating an electrode used for the valve and a $p \mathrm{H}$-sensitive electrode in a three-electrode system, the valve can be made $p \mathrm{H}$-responsive. A similar idea was used to construct a $p \mathrm{H}$-stat that maintained the $p \mathrm{H}$ of a solution at a constant value by a negative-feedback function. The $p \mathrm{H}$-stat could measure the amount of protons or hydroxyl ions produced during the process. Furthermore, a freeze-dried enzyme substrate was used to achieve rapid mixing to measure the initial rate of the reaction. By incorporating these techniques, a series of procedures could be carried out automatically. In this article, we present an example of a device with high functionalities introducing basic techniques related to this topic.

\section{BASIC TECHNIQUES TO CREATE MULTIFUNCTIONAL SYSTEMS}

\section{A. Electrowetting-based components for the control of microfluidic transport}

Sophisticated microfluidic systems require components that can control the transport of solutions precisely and reliably. The development of pumps and valves has been one of the critical themes in micromachining, and various designs have been proposed. ${ }^{7,8}$ Although the final goals of development depend on the applications, a critical point for the successful realization of such devices is the simplification of the structure and function of the components. To this end, electrowetting provides an effective solution. Electrowetting is a technique to change the wettability of a metal surface or dielectric on an electrode by controlling the electrode potential. $^{9,10}$ One of the promising applications of elec- 
trowetting is, indeed, microfluidic transport. Currently, many devices designed for the handling of droplets have been reported. ${ }^{11-18}$ In these devices, droplets are mobilized on an array of electrodes covered with a hydrophobic insulating layer by changing the wettability of the surface on one of the electrodes one by one. Although the new technology has resulted in the creation of a novel concept, i.e., digital microfluidics, combinations with other techniques, such as sampling and sensing, need further consideration. Furthermore, present chemical analyses, including those carried out on microfluidic devices, are based on the handling of continuous liquids. Therefore, our effort has been directed toward the development of devices for continuous liquids. To this end, we have mainly used gold electrodes that were in direct contact with a solution to generate a driving force or to function as a valve. A liquid column can be mobilized to desired directions in a network of branched flow channels. ${ }^{19-21} \mathrm{An}$ advantage with this approach is that the applied potential is very small $(\sim 1 \mathrm{~V})$, and transport of the solution can be controlled using a potentiostat widely used for electroanalysis. On the other hand, electrolysis that may occur at a larger overpotential is often claimed to be a disadvantage. Along with the ordinary use for microfluidic components, direct electrowetting has an advantage in that it facilitates the creation of automatic microsystems because the electrode system can be easily coupled with the electronic circuit of driving instruments. An example of such a function is a valve that opens automatically after two compartments separated by the valve are filled with solutions. ${ }^{22}$ On the basis of this idea, a valve that opens automatically in response to a solution $p \mathrm{H}$ can be constructed, an idea that we, in fact, incorporated in our present device.

Figure 1 shows the principle of operation of the $p \mathrm{H}$-responsive valve. The actual forms of the valve vary depending on the application. An electrode may be placed in a straight flow channel. Otherwise, as described in Fig. 3, an electrode may be formed on the wall of a through-hole. Here, to avoid confusion, the potential applied to the working electrode with respect to the $p \mathrm{H}$-sensitive reference electrode will be expressed as "voltage," whereas the potential of each electrode with respect to an assumed fixed potential standard (e.g., $\mathrm{Ag} / \mathrm{AgCl}$ ) will be referred to as "potential." For example, let us assume that a solution of a given $p \mathrm{H}$ is initially stopped and touches the edge of the working electrode (valve). Here, an appropriate negative voltage is applied to the working electrode with respect to the $p \mathrm{H}$-sensitive reference electrode [Fig. 1(a), top]. The potential is not sufficiently negative to cause electrowetting. When the $p \mathrm{H}$ of the solution is elevated, the potential of the $p \mathrm{H}$-sensitive reference electrode shifts to the negative side. Following this, a potential that exceeds the threshold value is applied to the working electrode [Fig. 1(a), bottom]. As a result, the wettability of the valve electrode changes, and the solution passes the valve area.

In the opposite case, in which the $p \mathrm{H}$ of the solution is lowered with a fixed voltage, the potential of the reference electrode and, simultaneously, the potential of the working electrode shift to the more positive side. However, with this change, causing reproducible changes is difficult, possibly (a)

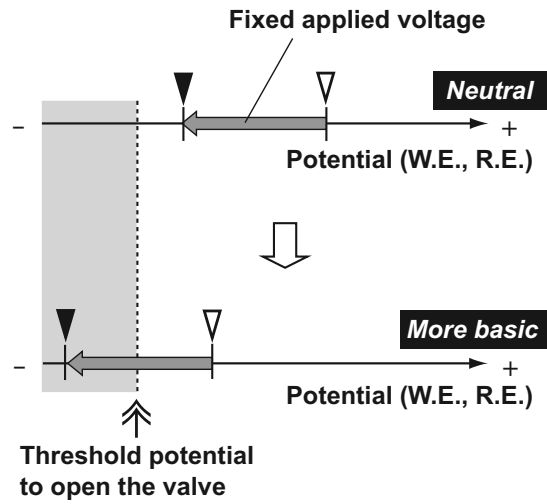

(b)

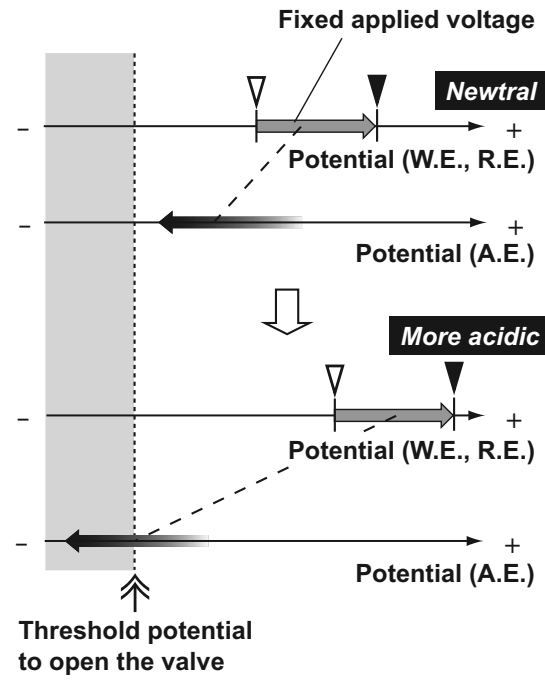

FIG. 1. Principle of operation of the $p \mathrm{H}$-responsive valve showing the procedure to open at a more basic $p \mathrm{H}$ (a) and a more acidic $p \mathrm{H}$ (b). The black and white triangles indicate the potential of the working electrode and the $p \mathrm{H}$-sensitive reference electrode measured with respect to a fixed potential standard, respectively. "Threshold potential" means the potential at which the valve opens. W.E., working electrode; R.E., reference electrode; and A.E., auxiliary electrode.

because of the specific adhesion of anions. To cope with the problem, an auxiliary electrode is used for the valve electrode [Fig. 1(b)]. When the potential of the working electrode shifts to the positive side, a small current is generated. As a result, the auxiliary electrode is polarized to the negative direction. As a result, the potential of the auxiliary electrode exceeds the threshold value, and the valve (in this case the auxiliary electrode) opens as in the previous case.

\section{B. Freeze-dried components for rapid mixing}

Mixing of components is a routine procedure in chemical analyses. In microfluidic systems, it is often carried out by flowing two streams of solutions into a microflow channel and mixing components by diffusion. Since the dimension of the flow channel normal to the flow is in the order of tens or hundreds of microns, small molecules mix relatively fast if the two-solution interface is sufficiently long or the stream is stopped. For larger molecules, microstructures have been used to disturb the flow and accelerate mixing. ${ }^{23-27}$ This approach, in which two streams of solutions are mixed, may be suitable for laboratory use. However, it produces an unwanted dead volume, resulting in an inefficient use of pre- 
(a)

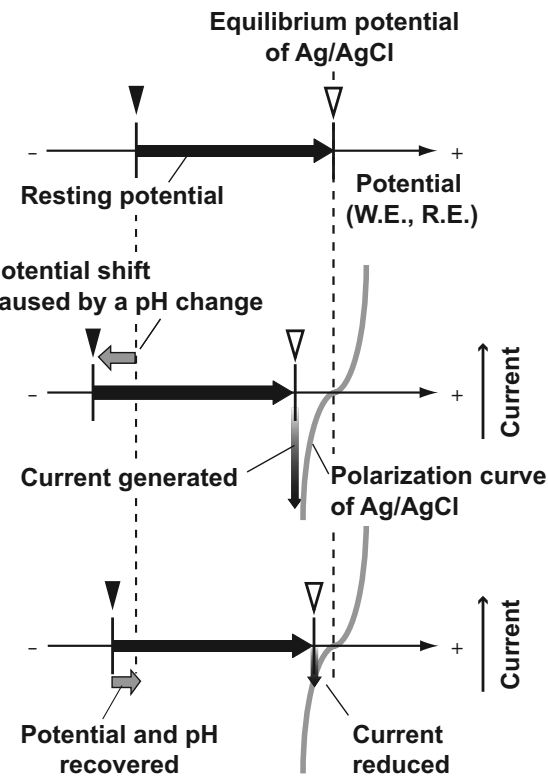

FIG. 2. Principle of operation of the $p \mathrm{H}$-stat. Responses of the electrodes to a slight $p \mathrm{H}$ change to the basic side are shown. (a) Resting potential corresponding to the $p \mathrm{H}$ is applied to the working electrode. (b) A large cathodic current is generated following the potential change in the $p \mathrm{H}$-sensitive reference electrode. (c) The potential of the reference electrode recovers to the initial value. The black and white triangles indicate the potentials of the $p \mathrm{H}$-sensitive reference electrode and the $\mathrm{Ag} / \mathrm{AgCl}$ working electrode measured with respect to a fixed potential standard, respectively. W.E., working electrode; R.E., reference electrode; and A.E., auxiliary electrode.

cious reagents. Moreover, micropumps must be integrated if the miniaturization of the entire system is required. An alternative approach is to use a valve to mix two solutions filled in two separate compartments. ${ }^{28}$ However, mixing of stationary solutions relies on diffusion alone, and rapid mixing becomes an issue. To solve these problems, a good alternative is to use freeze-dried components. ${ }^{29}$ In this approach, one group of components to be mixed is placed in the flow channel beforehand and dissolved into a solution containing the other group of components.

Freeze drying has been widely used for the preservation of materials ranging from foods to biochemical molecules. In the process of freeze drying, a solution containing the material is first frozen. The pressure is then reduced to allow the frozen water to sublime. Packing of a compartment in a microfluidic device with a freeze-dried matrix is easily achieved by allowing the solution to penetrate there and carrying out the same procedure. The solid components constitute only a part of the volume. Therefore, the matrix becomes extremely porous. The unique structure facilitates rapid penetration of solution and dissolution of the components.

\section{Electrochemical $p \mathrm{H}-\mathrm{stat}$}

Similarly to conventional chemical analysis, the analysis of biochemical compounds often requires the control of the $p \mathrm{H}$. As a representative case, the activity of an enzyme should be measured at a fixed $p \mathrm{H}$ because the activity is influenced by the $p \mathrm{H}$. The requirement becomes severer for hydrolytic enzymes because the reactions accompany local $p \mathrm{H}$ changes. To control the $p \mathrm{H}$, an ordinary method is to mix the solution to be handled with an acidic or a basic solution.

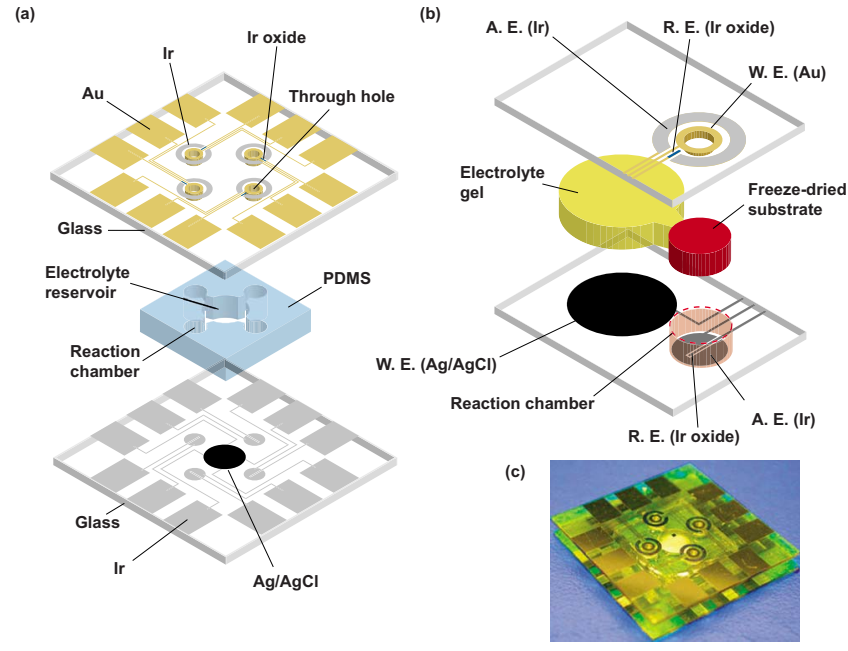

FIG. 3. (Color online) Microanalysis system for gastrointestinal information. (a) Decomposed structure. (b) Magnified view of one of the assay sites. (c) Completed system with four assay sites. W.E., working electrode; R.E., reference electrode; and A.E., auxiliary electrode.

However, this requires an additional injection mechanism. In this respect, titration using an electrochemical $p \mathrm{H}$-stat is more suitable in that the device can maintain the solution $p \mathrm{H}$ and, at the same time, results are obtained directly by electric signals.

We used an electrochemical $p \mathrm{H}$-stat based on the unique use of a three-electrode system. ${ }^{30}$ Figure 2 shows the principle of operation. Unlike the ordinary electrochemical threeelectrode system, a $p \mathrm{H}$-sensitive electrode is used for the reference electrode, and a nonpolarizable electrode for the working electrode. The auxiliary electrode is used as an actuator electrode to cause a $p \mathrm{H}$ change in a reaction chamber. A negative-feedback function maintains the $p \mathrm{H}$ of the solution at a fixed value. Here, let us suppose that a solution of a given $p \mathrm{H}$ is filled in the reaction chamber. The resting potential of the working electrode with respect to the reference electrode is measured and is fixed by using a potentiostat. When the solution $p \mathrm{H}$ in the reaction chamber changes, an overpotential is automatically applied to the working electrode following the potential change in the $p \mathrm{H}$-sensitive reference electrode. However, even with a very small potential change, a large current is generated because the electrode is nonpolarizable. Accompanying the current generation, the electrolysis of water occurs on the auxiliary electrode that surrounds the $p \mathrm{H}$-sensitive reference electrode. As a result, the solution $p \mathrm{H}$ recovers to the initial value, resulting in a decrease in the overpotential. The $p \mathrm{H}$ to be fixed can be changed by changing the potential of the working electrode with respect to the reference electrode. The basic components of the $p \mathrm{H}$-stat are the electrode patterns. Therefore, the entire device can be easily miniaturized and integrated with other components.

\section{PROTOPYPE OF A MULTIFUNCTIONAL DEVICE FOR THE ACQUISITION OF GASTROINTESTINAL INFORMATION}

Figure 3(a) shows a proof-of-concept device with four sensing sites. The three components reported earlier, namely, 


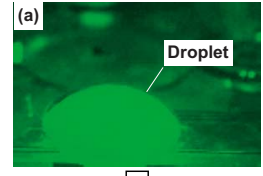

\3.
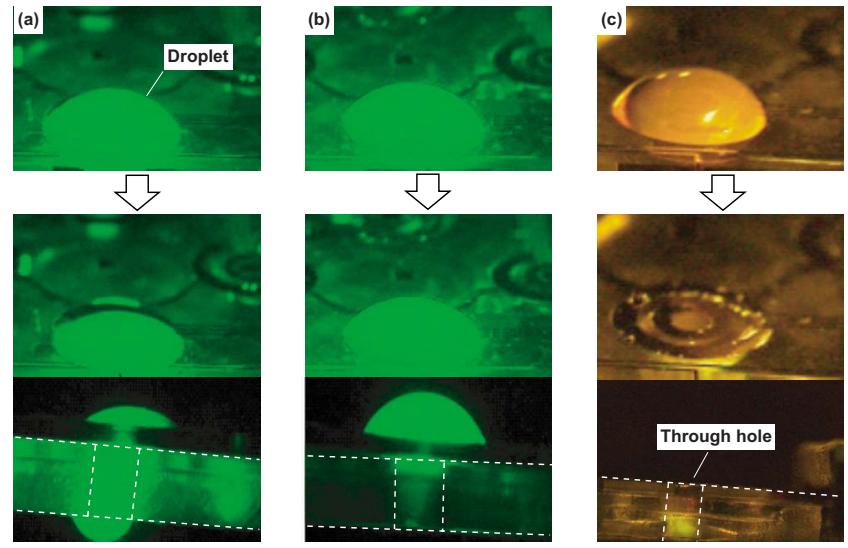

\3

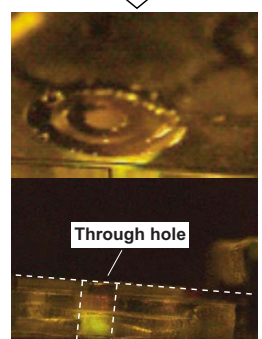

FIG. 4. (Color online) Movements of solutions placed in the valve. (a) -0.8 $\mathrm{V}$ was applied, and a solution of $p \mathrm{H} 8.0$ passed the valve area. (b) $-0.8 \mathrm{~V}$ was applied, but a solution of $p \mathrm{H} 7.2$ did not pass the valve area. (c) $+0.6 \mathrm{~V}$ was applied, and a solution of $p \mathrm{H} 2.0$ passed the valve area. In (c), the auxiliary electrode was used as the valve. The figures at the bottom are side views. The following buffer solutions were used: $0.1 M \mathrm{KCl}-\mathrm{HCl}(p \mathrm{H} \mathrm{2.0})$, $25 \mathrm{mM} \mathrm{KH} \mathrm{PO}_{4}-\mathrm{NaOH}(p \mathrm{H} \mathrm{7.2})$, and $50 \mathrm{mM}$ tris- $\mathrm{HCl}(p \mathrm{H} \mathrm{8.0})$.

a $p \mathrm{H}$-responsive valve, a freeze-dried enzyme substrate, and a $p \mathrm{H}$-stat, were placed in each sensing site. Compartments were formed with polydimethylsiloxane (PDMS). For this purpose, a template for the microstructures was first formed with a thick-film photoresist, SU-8 (MicroChem, Newton, MA), on a glass substrate. Then, precursor solutions for PDMS (KE-1300T and CAT-1300, Shin-Etsu Chemical, Tokyo, Japan) were mixed well and poured onto the template. After PDMS was cured, it was removed from the template, and a faithful reproduction of the template structure was obtained.

The electrodes were formed on a glass chip (Corning No. 7740, $500 \mu \mathrm{m}$ thick) by a thin-film process including sputtering. The three-electrode system used for the operation consisted of a gold electrode that worked as a valve, an iridium oxide $p \mathrm{H}$-sensitive reference electrode, and an iridium auxiliary electrode. A through-hole (diameter: $1 \mathrm{~mm}$ ) was formed in the glass chip by mechanical drilling, and the gold electrode was formed on the wall of the through-hole (Fig. 4). Gold and iridium electrodes were used as the working and auxiliary electrodes, respectively, and their role was changed depending on the direction of the $p \mathrm{H}$ change. For the $p \mathrm{H}$-sensitive electrode, any potentiometric $p \mathrm{H}$-electrode can be used. A variety of $p \mathrm{H}$ sensors have been proposed, including metal oxide sensors, sensors with ionophores, and ion-sensitive field effect transistors. ${ }^{31,32}$ From among them, we selected iridium oxide grown by electrochemical potential cycling ${ }^{33}$ because the structure and fabrication of the electrode are simple. In forming the film, the iridium pattern was immersed in a $1 M \mathrm{LiClO}_{4}$ solution, and a triangular voltage waveform was applied $[-0.9$ to $+1.2 \mathrm{~V}$ (versus $\mathrm{Ag} /$ $\mathrm{AgCl}), 0.5 \mathrm{~V} / \mathrm{s}]$ using a function generator (HB-111, Hokuto Denko, Tokyo, Japan) connected to a potentiostat. ${ }^{34}$

The $p \mathrm{H}$-stat used a different type of nonstandard threeelectrode system reported earlier, which was formed on the other glass chip. An iridium oxide $p \mathrm{H}$-sensitive electrode was used for the reference electrode and placed in a reaction chamber. The reference electrode was surrounded by an iri- dium auxiliary electrode that worked as an actuator electrode to cause the electrolysis of water. $\mathrm{A} \mathrm{Ag} / \mathrm{AgCl}$ electrode was used for the working electrode and placed in a separate compartment in contact with an electrolyte gel. A AgCl layer had been grown electrochemically on the silver layer. The areas of the reference, working, and auxiliary electrodes were rectangular or circular and $900 \times 50 \mu^{2}, 3.0 \mathrm{~mm}$, and $1.6 \mathrm{~mm}$ in diameter, respectively; all of them were delineated with a polyimide insulating layer (Semicofine SP-341, Toray, To-

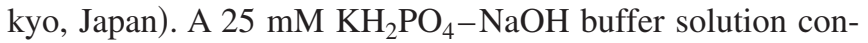
taining $0.1 M \mathrm{KCl}(p \mathrm{H} 7.2)$ impregnated in a gel was used as an internal electrolyte for the $\mathrm{Ag} / \mathrm{AgCl}$ working electrode. The gel was formed by photopolymerization of a precursor solution of PVA-SbQ (SPP-H-13, Toyo Gosei Kogyo, Chiba, Japan).

A freeze-dried substrate layer was formed in the reaction chamber. Here, bovine serum albumin (BSA) (SigmaAldrich, St. Louis, MO) or bovine hemoglobin (Merck Calbiochem, San Diego, CA) was used as a substrate for the proteases. When using BSA, a 5 wt $\%$ BSA solution was filled in the reaction chamber and frozen at $-80{ }^{\circ} \mathrm{C}$ for 45 min followed by drying for $30 \mathrm{~min}$ in a vacuum using a freeze drier (FDU-1200, Eyela, Tokyo, Japan). When using hemoglobin, a 2 wt \% solution was filled in the chamber, and the same procedure was followed. The porous structure was observed using a scanning electron microscope (TM1000, Hitachi High-Technologies, Tokyo, Japan).

The potential of the working electrode for the $p \mathrm{H}$-responsive valve was controlled using a potentiostat (HA-151, Hokuto Denko, Tokyo, Japan). The progress of the enzymatic reactions was analyzed using an Autolab PGSTAT12 (Eco Chemie, Utrecht, Netherlands).

\section{CHARACTERIZATION OF THE PERFORMANCE}

\section{A. Microfluidic components}

Electrowetting-based valves have been used in our devices to carry out on-chip analyses. ${ }^{22,35}$ This simple structure facilitated the integration of many valves. Although previous devices have relied on manual switching, the use of the nonstandard electrode system resulted in the design of a valve that sensed the $p \mathrm{H}$ of a solution and opened. The threshold $p \mathrm{H}$ at which the valve opened could be adjusted by changing the working electrode potential with respect to the reference electrode. Figure 4 shows how the valve responded to solutions of different $p \mathrm{Hs}$. The fluorescence images were acquired with a fluorescence microscope (VB-G25, Keyence, Osaka, Japan) equipped with a charge-coupled device detection system (VB-7000/7010, Keyence). Prior to injection, an appropriate potential had been applied to the working electrode. Immediately after a solution of $p \mathrm{H} 8.0$ was placed in the valve area, the solution passed the through-hole spontaneously [Fig. 4(a)]. On the other hand, when a solution of $p \mathrm{H} 7.2$ was used, the circular valve area remained hydrophobic, and the surface tension kept the solution from dropping

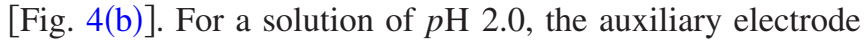
was used as the valve electrode. The valve responded in a similar manner. A solution of $p H 2.0$ passed the valve, whereas a solution of $p \mathrm{H} 7.2$ was blocked from passing it. 
(a)

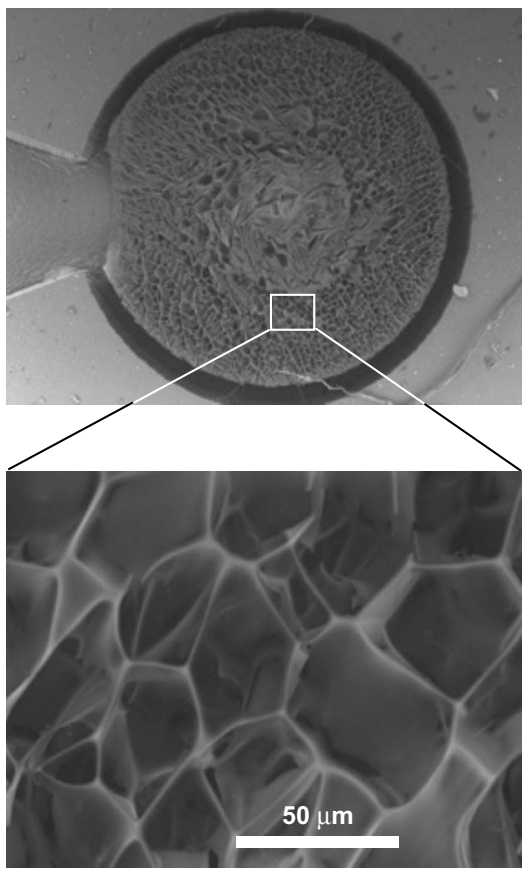

(b)

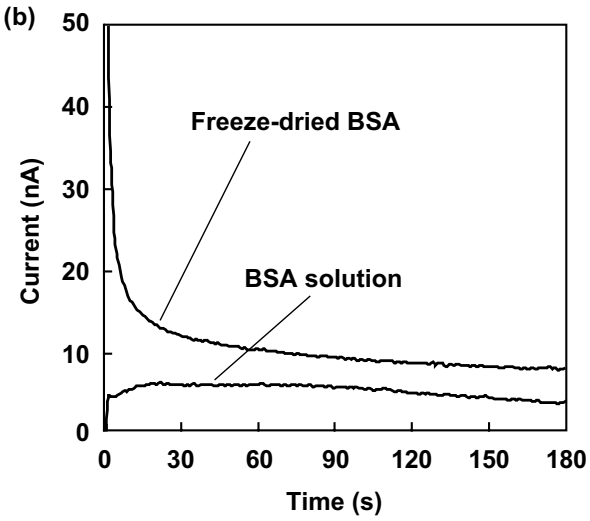

FIG. 5. Scanning electron microscope images of the freeze-dried enzyme substrate (a) and variation of the output current after the injection of a trypsin solution (b). The activity of trypsin was $267 \mathrm{U} / \mathrm{ml}$. The trypsin standard solution was prepared with a $50 \mathrm{mM}$ tris- $\mathrm{HCl}$ buffer solution containing $50 \mathrm{mM} \mathrm{CaCl}_{2}\left(p \mathrm{H} \mathrm{8.0)}\right.$. The measurements were conducted at $37{ }^{\circ} \mathrm{C}$.

The present $p \mathrm{H}$-responsive valves open at $p \mathrm{Hs}$ higher or lower than a threshold value. In this sense, they are not completely $p \mathrm{H}$-selective. However, by combining the two types of valves, a $p \mathrm{H}$ filter that passes only a solution of a narrow $p \mathrm{H}$ range will be realized.

\section{B. Effect of the freeze-dried substrate}

When measuring enzyme activity, a critical indicator is the initial rate of the reaction because the rate decreases as the enzyme substrate becomes depleted. Therefore, rapid instantaneous mixing becomes particularly important. Since the freeze-dried substrate occupied the entire volume of the reaction chamber and was extremely porous [Fig. 5(a)], it dissolved rapidly after the solution penetrated into the matrix. The effect was examined in the hydrolysis of BSA by trypsin (EC 3.4.21.4, from bovine pancreas, $13500 \mathrm{U} / \mathrm{mg}$, Sigma-Aldrich) using only the $p \mathrm{H}$-stat. The trypsin standard solution was prepared with a $50 \mathrm{mM}$ tris- $\mathrm{HCl}$ buffer solution containing $50 \mathrm{mM} \mathrm{CaCl}_{2}(p \mathrm{H} \mathrm{8.0)}$. The activities were also checked by the Schwert method. ${ }^{36}$ We first conducted a comparative experiment by filling a BSA solution in the reaction chamber and adding the enzyme solution. A predetermined potential had been applied to the working electrode for the $p \mathrm{H}$-stat prior to mixing. Although the thickness of the underlying solution was only $0.8 \mathrm{~mm}$ and the impact should have caused a physical disturbance, the time required for mixing was not negligible. In fact, the variation of the measured current was small, suggesting that the enzymatic reaction proceeded in parallel with gradual mixing [Fig. 5(b)]. In addition, the current was small because of unavoidable dilution. On the other hand, when the freeze-dried substrate was used, a charging current was observed when the solution wetted the electrode, and the current decreased monotonically thereafter. Furthermore, the generated current was much larger than it was when the substrate solution was used [Fig. 5(b)]. The result suggests that the freeze-dried enzyme substrate dissolved rapidly and depleted with the progress of the enzymatic reaction.

\section{From sampling to the analysis of protease activities}

In measuring the activity of a hydrolytic enzyme, a problem is the deviation of the $p \mathrm{H}$ from an optimum value accompanying the hydrolytic reaction. The $p \mathrm{H}$-stat measures the amount of protons or hydroxyl ions produced during the enzymatic reaction, maintaining the $p \mathrm{H}$ at the optimum value. Sequential processing on the chip from the introduction of a standard solution to the measurement of the activity was checked using trypsin and pepsin (EC 3.4.23.1, from porcine stomach mucosa, $3370 \mathrm{U} / \mathrm{mg}$, Sigma-Aldrich). The enzymes hydrolyze peptide bonds and change the solution $p \mathrm{H}$ to the acidic side or the basic side, respectively. The amount of protons or hydroxyl ions produced accompanying the enzymatic reaction is known as the measured electric charge. Therefore, the rate of the enzymatic reaction, or the activity of the enzyme, is directly related to the output current.

The activity was measured at the optimum $\mathrm{pH}$ of the enzymes (8.0 for trypsin and 2.0 for pepsin) using freezedried hemoglobin as the enzyme substrate. The activity of trypsin and pepsin was also measured using conventional UV-spectrophotometric methods. The former was that reported earlier, and the latter was based on the Anson method. $^{37}$ After the injection of each enzyme, the $p \mathrm{H}$-stat was switched on, and the generated current was measured. Clear linear relationships were observed between the current and the activity of trypsin [Fig. 6(a)] or pepsin [Fig. 6(b)].

Although the size of the present chip is not small (20 $\times 20 \mathrm{~mm}^{2}$ ), a substantial portion is occupied by dead space, and further miniaturization is indeed possible. If another module with integrated circuits is incorporated for wireless signal transmission, the present technology will enable the acquisition of gastrointestinal information. 
(a)

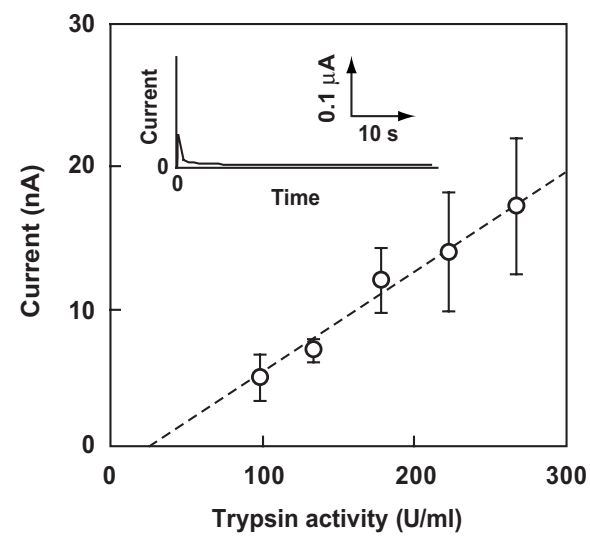

(b)

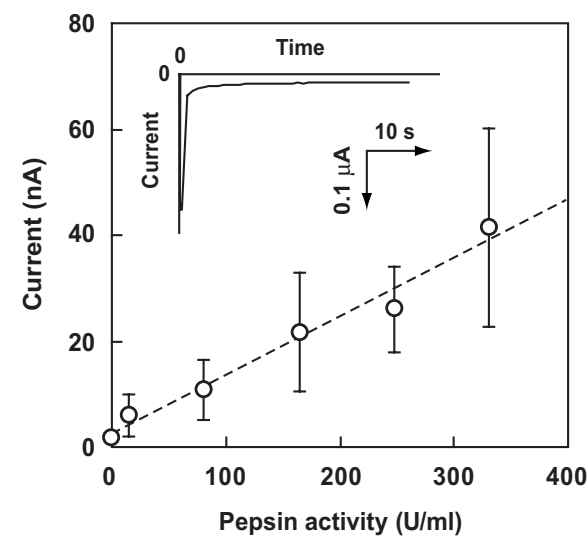

FIG. 6. Dependence of the initial current on the activity of trypsin (a) and pepsin (b). The current at $5 \mathrm{~s}$ was plotted. Five runs were made, and averages and standard deviations are shown. The insets show the time courses of the output current. Time 0 indicates the point at which a trypsin standard solution $(267 \mathrm{U} / \mathrm{ml})$ or a pepsin standard solution $(331 \mathrm{U} / \mathrm{ml})$ was injected The standard solutions were prepared with buffer solutions of the same $p \mathrm{Hs}$ (50 $\mathrm{mM}$ tris- $\mathrm{HCl}$ for trypsin and $0.1 \mathrm{M} \mathrm{KCl}-\mathrm{HCl}$ for pepsin). The measurements were conducted at $37^{\circ} \mathrm{C}$.

\section{POSSIBILITY OF INCORPORATING OTHER SENSORS}

In the fabricated device, we used sampling, $p \mathrm{H}$-regulation, and sensing functions to measure the activity of proteases. However, for gastrointestinal monitoring or other applications, simultaneous measurement of analytes, such as the $p \mathrm{H}$, is often required. The determination of chemical or biological molecules of low molecular weights has been attempted using miniaturized devices, and remarkable progress in the use of this technique has been made over the past decade. ${ }^{32,38}$ Therefore, the incorporation of the sensors in our present device will be easily realized, as demonstrated in our previous work. ${ }^{39}$ On the other hand, the detection of DNAs and proteins needs further consideration because the analysis of these molecules requires sequential reactions accompanying the exchange of many solutions. Although we avoided the use of micropumps in our device, they will be necessary in any form. Furthermore, a certain amount of solution used for cleansing will be necessary.

\section{CONCLUSION}

An integrated microanalysis system for the determination of the activity of proteases can be realized by using a $p \mathrm{H}$-responsive valve and a $p \mathrm{H}$-stat. The $p \mathrm{H}$-responsive valve is fabricated on the basis of electrowetting using a nonstandard three-electrode system. The $p \mathrm{H}$-stat is constructed by using another nonstandard three-electrode system. The $p \mathrm{H}$-responsive valve functions at a lower or higher $p \mathrm{H}$, and the necessary solution can be introduced into the sensing area. Freeze-dried enzyme substrates promote rapid dissolution and mixing. Therefore, the response performance is significantly better than that of the conventional method based on solution mixing, particularly for the analysis of enzyme activity. The determination of the activity of trypsin and pepsin can be conducted by measuring the charge generated accompanying the hydrolytic reactions at their optimum $p \mathrm{Hs}$. A series of procedures from sampling to the measurement of enzyme activity can be carried out using this system.

\section{ACKNOWLEDGMENTS}

This study was supported by a Grant-in-Aid for Scientific Research (B) (Grant No. 19360369) from the Japan Society for the Promotion of Science.

${ }^{1}$ A. Z. Al-Bahrani and B. J. Ammori, Clin. Chim. Acta 362, 26 (2005).

${ }^{2}$ R. E. Ionescu, S. Cosnier, and R. S. Marks, Anal. Chem. 78, 6327 (2006).

${ }^{3}$ A. Moglia, A. Menciassi, M. O. Schurr, and P. Dario, Biomed. Microdevices 9, 235 (2007).

${ }^{4}$ G. Iddan, G. Meron, A. Glukhovsky, and P. Swain, Nature (London) 405, 417 (2000)

${ }^{5}$ E. A. Johannessen, L. Wang, S. W. J. Reid, D. R. S. Cumming, and J. M. Cooper, Lab Chip 6, 39 (2006).

${ }^{6}$ T. B. Tang, E. A. Johannessen, L. Wang, A. Astaras, M. Ahmadian, A. F. Murray, J. M. Cooper, S. P. Beaumont, B. W. Flynn, and D. R. S. Cumming, IEEE Sens. J. 2, 628 (2002).

${ }^{7}$ G. T. A. Kovacs, Micromachined Transducers Sourcebook (WCB McGraw-Hill, Boston, MA, 1998), pp. 823-855.

${ }^{8}$ D. J. Laser and J. G. Santiago, J. Micromech. Microeng. 14, R35 (2004).

${ }^{9}$ C. Quilliet and B. Berge, Curr. Opin. Colloid Interface Sci. 6, 34 (2001).

${ }^{10}$ F. Mugele and J.-C. Baret, J. Phys.: Condens. Matter 17, R705 (2005).

${ }^{11}$ M. G. Pollack, R. B. Fair, and A. D. Shenderov, Appl. Phys. Lett. 77, 1725 (2000)

${ }^{12}$ M. G. Pollack, A. D. Shenderov, and R. B. Fair, Lab Chip 2, 96 (2002).

${ }^{13}$ H. Moon, S. K. Cho, R. L. Garrell, and C.-J. Kim, J. Appl. Phys. 92, 4080 (2002).

${ }^{14}$ P. Paik, V. K. Pamula, and R. B. Fair, Lab Chip 3, 253 (2003).

${ }^{15}$ P. Y. Chiou, H. Moon, H. Toshiyoshi, C. J. Kim, and M. C. Wu, Sens. Actuators, A 104, 222 (2003).

${ }^{16}$ V. Srinivasan, V. K. Pamula, and R. B. Fair, Anal. Chim. Acta 507, 145 (2004).

${ }^{17}$ I. Moon and J. Kim, Sens. Actuators, A 130-131, 537 (2006).

${ }^{18}$ V. Srinivasan, V. K. Pamula, and R. B. Fair, Lab Chip 4, 310 (2004).

${ }^{19}$ W. Satoh, M. Loughran, and H. Suzuki, J. Appl. Phys. 96, 835 (2004).

${ }^{20}$ W. Satoh, H. Yokomaku, H. Hosono, N. Ohnishi, and H. Suzuki, J. Appl. Phys. 103, 034903 (2008).

${ }^{21}$ W. Satoh, H. Hosono, and H. Suzuki, Anal. Chem. 77, 6857 (2005).

${ }^{22}$ H. Hosono, W. Satoh, M. Toya, K. Morimoto, J. Fukuda, and H. Suzuki, Sens. Actuators B 132, 614 (2008).

${ }^{23}$ F. G. Bessoth, A. J. deMello, and A. Manz, Anal. Commun. 36, 213 (1999).

${ }^{24}$ R. H. Liu, M. A. Stremler, K. V. Sharp, M. G. Olsen, J. G. Santiago, R. J. Adrian, H. Aref, and D. J. Beebe, J. Microelectromech. Syst. 9, 190 (2000).

${ }^{25}$ A. D. Stroock, S. K. W. Dertinger, A. Ajdari, I. Mezić, H. A. Stone, and G. M. Whitesides, Science 295, 647 (2002).

${ }^{26}$ D. J. Kim, H. J. Oh, T. H. Park, J. B. Choo, and S. H. Lee, Analyst (Lond.) 130, 293 (2005)

${ }^{27}$ A. P. Sudarsan and V. M. Ugaz, Proc. Natl. Acad. Sci. U.S.A. 103, 7228 (2006).

${ }^{28}$ K. Morimoto and H. Suzuki, Biosens. Bioelectron. 22, 86 (2006).

${ }^{29}$ K. Morimoto, S. Upadhyay, T. Higashiyama, N. Ohgami, H. Kusakabe, J. 
Fukuda, and H. Suzuki, Sens. Actuators B 124, 477 (2007).

${ }^{30}$ K. Morimoto, M. Toya, J. Fukuda, and H. Suzuki, Anal. Chem. 80, 905 (2008).

${ }^{31}$ P. Bergveld, Sens. Actuators B 88, 1 (2003).

${ }^{32}$ H. Suzuki, S. Upadhyay, and M. Loughran, in Encyclopedia of Sensors, edited by C. A. Grimes, E. C. Dickey, and M. V. Pishko (American Scientific, Stevenson Ranch, CA, 2006), Vol. 6, pp. 161-187.

${ }^{33}$ M. L. Hitchman and S. Ramanathan, Analyst (Lond.) 113, 35 (1988).

${ }^{34}$ H. Suzuki, H. Shiroishi, S. Sasaki, and I. Karube, Anal. Chem. 71, 5069 (1999).
${ }^{35}$ N. Nashida, W. Satoh, J. Fukuda, and H. Suzuki, Biosens. Bioelectron. 22, 3167 (2007).

${ }^{36} \mathrm{H}$. U. Bergmeyer, in Methods of Enzymatic Analysis, 2nd ed., edited by $\mathrm{H}$. U. Bergmeyer (Academic, New York, 1974), Vol. 1, pp. 515-516.

${ }^{37}$ W. Rick and W.-P. Fritsch, in Methods of Enzymatic Analysis, 2nd ed., edited by H. U. Bergmeyer (Academic, New York, 1974), Vol. 2, pp. 1046-1057.

${ }^{38} \mathrm{H}$. Suzuki, Electroanalysis 12, 703 (2000).

${ }^{39}$ W. Satoh, H. Hosono, H. Yokomaku, K. Morimoto, S. Upadhyay, and H. Suzuki, Sensors 8, 1111 (2008). 